\title{
Effectively Detecting Left Bundle Branch Block False Defects in Myocardial Perfusion Imaging (MPI) with a Convolutional Neural Network (CNN)
}

\author{
Mohamed E. H. ABDI ${ }^{\mathrm{a}, 1}$ Qais NAILI ${ }^{\mathrm{a}}$, Mahdi HABBACHE ${ }^{\mathrm{a}}$, Bilhadj SAID ${ }^{\mathrm{a}}$, \\ Abdelhak BOUMENIR ${ }^{\mathrm{a}}$, Taouba DOUIBI ${ }^{\mathrm{a}}$, Dahlia DJERMANE ${ }^{\mathrm{b}}$ and \\ Sid-Ahmed BERRANI ${ }^{\mathrm{C}}$ \\ ${ }^{\text {a } C e n t r e ~ d ' i m a g e r i e ~ s c i n t i g r a p h i q u e, ~ B l i d a, ~ A l g e r i a ~}$ \\ ${ }^{\mathrm{b}}$ Cardio A2, CHU Mustapha Bacha, Algiers, Algeria \\ ${ }^{\mathrm{c}}$ National Polytechnic School, Algiers, Algeria
}

\begin{abstract}
Left bundle branch block (LBBB) is a frequent source of false positive MPI reports, in patients evaluated for coronary artery disease. Purpose: In this work, we evaluated the ability of a CNN-based solution, using transfer learning, to produce an expert-like judgment in recognizing LBBB false defects. Methods: We collected retrospectively, MPI polar maps, of patients having small to large fixed anteroseptal perfusion defect. Images were divided into two groups. The LBBB group included patients where this defect was judged as false defect by two experts. The LAD group included patients where this defect was judged as a true defect by two experts. We used a transfer learning approach on a CNN (ResNet50V2) to classify the images into two groups. Results: After 60 iterations, the reached accuracy plateau was 0.98 , and the loss was 0.19 (the validation accuracy and loss were 0.91 and 0.25 , respectively). A first test set of 23 images was used (11 LBBB, and $12 \mathrm{LAD}$ ). The empiric ROC (Receiver operating characteristic) Area was estimated at 0.98 . A second test set (18x2 images) was collected after the final results. The ROC area was estimated again at 0.98. Conclusion: Artificial intelligence, using $\mathrm{CNN}$ and transfer learning, could reproduce an expert-like judgment in differentiating between LBBB false defects, and LAD real defects.
\end{abstract}

Keywords. left bundle branch block, obstructive coronary artery disease, GATED SPECT myocardial perfusion imaging, convolutional neural network

\section{Introduction}

Left bundle branch block (LBBB) is a frequent source of false positive reports in myocardial perfusion imaging (MPI). This has been reported in previous studies that have evaluated the relation between myocardial perfusion and LBBB $[1,2]$.

The method of interpretation used in MPI in patients having LBBB, may influence the sensitivity and specificity of the exam. In this paper by Higgins et al. [1], authors concluded that the use of certain features of the MPI scan can aid the clinician in differentiating true perfusion defects, distinguishing underlying ischemia from false

\footnotetext{
${ }^{1}$ Corresponding Author, M. ABDI, CIS Blida (scintigraphies.com); E-mail: dr.abdi.m.h@gmail.com
} 
defects. We try to evaluate the usefulness of artificial intelligence, to reproduce this method of interpretation, offering a perspective to develop a diagnostic aid tool.

Some previous studies have evaluated the value of deep learning in the diagnosis of coronary artery disease (CAD) in MPI [3, 4, 5]. Betancur J et al. [3] concluded that deep learning improves automatic prediction of obstructive coronary artery disease from MPI, as compared to the current standard quantitative method.

However, such studies did not include some clinical information when training the model, such as for example, the existence of a LBBB.

\section{Purpose}

In this work, we evaluated the ability of a CNN based solution, using transfer learning, to produce an expert-like judgment in differentiating LBBB false defect, from left anterior descending artery (LAD) real perfusion defect. The study was conducted considering a small dataset, because collecting a larger dataset needs a proof of utility.

\section{Materials and Methods}

\subsection{Study population}

The study covered two groups of MPI polar map images, collected retrospectively from our department, with small, to large fixed antéroseptal perfusion defect (small: 1 segment, moderate: 2 segments, large: 3 or more segments, based on the 17 segments model [6]).

- $\quad$ The LBBB group included patients where the perfusion defect was judged as false defect by two experts (based on clinical assessment, and GATEDSPECT [1]). Expert judgment was reinforced by a flow up for 3 years (no cardiovascular event). All patients in this group had a LBBB.

- The LAD group included patients where the perfusion defect was judged as a true positive by two experts. Expert judgment was confirmed by angiography; $>70 \%$ narrowing of LAD artery (patients with more than one vessel disease, or LBBB on ECG, were excluded from this group).

Study population baseline characteristics, are illustrated in table1

Table 1. Baseline Characteristics of Studied Population

\begin{tabular}{rrrrr}
\hline Characteristic & Overall $\mathbf{n}=\mathbf{6 3}$ & $\begin{array}{c}\text { LBBB } \mathbf{n}=\mathbf{3 3} \\
\mathbf{( 5 2 . 3 8 \% )}\end{array}$ & $\begin{array}{r}\text { LAD } \mathbf{n}=\mathbf{3 0} \\
\mathbf{( 4 7 . 6 2 \% )}\end{array}$ & $\boldsymbol{P}$ \\
\hline Age & $63.08 \pm 2.46$ & $64.18 \pm 3.44$ & $61.82 \pm 3.46$ & 0.0087 \\
Male & $26(41.27 \%)$ & $5(15.15 \%)$ & $21(70.00 \%)$ & $<0.0001$ \\
Female & $37(58.73 \%)$ & $28(84.84 \%)$ & $9(30.00 \%)$ & 0.0001 \\
Hypertension & $35(55.55 \%)$ & $21(63.63 \%)$ & $14(46.66 \%)$ & 0.1793 \\
Diabetes & $21(33.33 \%)$ & $11(33.33 \%)$ & $10(33.33 \%)$ & 1.0000 \\
Dyslipidemia & $6(9.52 \%)$ & $4(12.12 \%)$ & $2(6.66 \%)$ & 0.4644 \\
Smoking & $7(11.11 \%)$ & $0(0 \%)$ & $7(23.33 \%)$ & 0.0035 \\
Chest pain & $30(47.61 \%)$ & $20(60.60 \%)$ & $10(33.33 \%)$ & 0.0318 \\
Dyspnea & $4(6.34 \%)$ & $4(12.12 \%)$ & $0(0 \%)$ & 0.0506 \\
\hline
\end{tabular}




\subsection{Image Acquisition}

A conventional single head, Gamma Camera was used for all patients, using Tc99mSestamibi radiotracer. Patients had various stress protocols, such as treadmill, pharmacological, or a mixed protocol. Stress and rest exams were performed either the same day, or on two different days.

\subsection{Images Dataset}

The dataset was composed of 107 perfusion polar maps (42 images in each class for training, with $29 \%$ for validation). Stress and rest images were used.

\subsection{Deep learning}

Several CNN were tested, and ResNet50V2 was chosen for achieving the best results.

Only the classification part of the network was re-trained, following a transfer learning approach (training a fully connected layer, with two neurons).

\section{Results}

After 60 iterations, the reached accuracy plateau was 0.98 , and the loss was 0.19 (the validation accuracy and loss were 0.91 and 0.25 , respectively).

A first test set of 23 images was used (11 LBBB, and $12 \mathrm{LAD})$. The empiric Receiver operating characteristic (ROC) Area was estimated at 0.98 , with $95.7 \%$ accuracy.

A second test set (18 images in each group) was collected after the final results (but without the 3 years follow-up for the LBBB group). The ROC area for the model, was estimated again at 0.98 .

An example of a man of 70 years old, smoking, having a rest angina, and a LBBB on ECG, is illustrated (Figure 1). He had a positive stress, with reduced LVEF at 35\%. Images of this patient were not used nor in training, nor in validation. Stress image was predicted by our model as an LAD real perfusion defect. The rest image was predicted as a LBBB false defect, which means that according to our model, this patient is having an ischemia in the LAD territory (Figure 1). This patient had an angiography, confirming a severe narrowing of proximal LAD. He had a revascularization, resulting in an improvement of his LVEF (from $35 \%$ to $45 \%$ ).

This example illustrates the capacity of the model, differentiating real from false defect, in a same patient having CAD in the LAD artery, along with LBBB.

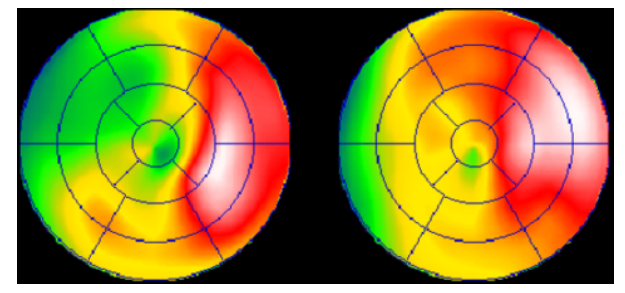

Figure 1. Example case. Left is the Stress polar map, predicted as a real LAD perfusion defect, and right is Rest image predicted as LBBB false defect. 


\section{Discussion}

\subsection{Study population}

It is worth clarifying that the two groups used in our study are not of the same cardiovascular risk level, as long as we are looking for false defects in the LBBB group, and real defects in the LAD group. We believe that such contrast is mandatory to train the model on distinct features from each group.

\subsection{Limitations}

Even if these results are encouraging, this study is still a retrospective one, done on a small number of patients, from a single department. Also, it has to be said that our model was trained on a specific color map used in our department, so the evaluation of the model on other patients from other departments needs to convert images to this specific color map.

The aim of this study was to evaluate the ability of deep learning to reproduce an expert like judgment for this problem. The next step could be a multicentric study (different gamma cameras), with coronary angiography as ground truth.

\section{Conclusion}

Artificial intelligence, using $\mathrm{CNN}$ and transfer learning -even on a very small training dataset- could reproduce an expert-like judgment in differentiating between LBBB false defect and LAD real perfusion defect. These results are motivating for a multicenter prospective study, to develop a diagnostic aid tool for clinicians, offering an expert like lecture. Such tool, could probably reduce false positive MPI reports, and by the way, reduce the number of unnecessary invasive angiography.

\section{References}

[1] Higgins JP, Williams G, Nagel JS, Higgins JA. Left bundle-branch block artifact on single photon emission computed tomography with technetium Tc $99 \mathrm{~m}$ (Tc-99m) agents: mechanisms and a method to decrease false-positive interpretations. Am Heart J. 2006;152(4):619-626.

[2] Gupta K, Bajaj NS, Hage FG, Bhambhvani P. Myocardial perfusion artifacts in left bundle branch block: A diagnostic challenge [published online ahead of print, 2019 May 2]. J Nucl Cardiol. 2019;10.1007/s12350-019-01717-9.

[3] Betancur J, Commandeur F, Motlagh M, et al. Deep Learning for Prediction of Obstructive Disease From Fast Myocardial Perfusion SPECT: A Multicenter Study. JACC Cardiovasc Imaging. 2018;11(11):1654-1663.

[4] Betancur J, Hu LH, Commandeur F, et al. Deep Learning Analysis of Upright-Supine High-Efficiency SPECT Myocardial Perfusion Imaging for Prediction of Obstructive Coronary Artery Disease: A Multicenter Study. J Nucl Med. 2019;60(5):664-670.

[5] Apostolopoulos ID, Papathanasiou ND, Spyridonidis T, Apostolopoulos DJ. Automatic characterization of myocardial perfusion imaging polar maps employing deep learning and data augmentation. Hell $\mathrm{J}$ Nucl Med. 2020;23(2):125-132.

[6] Cerqueira MD, Weissman NJ, Dilsizian V, Jacobs AK, Kaul S, Laskey WK, Pennell DJ, Rumberger JA, Ryan T, Verani MS; American Heart Association Writing Group on Myocardial Segmentation and Registration for Cardiac Imaging. Standardized myocardial segmentation and nomenclature for tomographic imaging of the heart. Circulation. 2002 Jan 29;105(4):539-42. 